\title{
Rôle du virus de l'hépatite C (HCV) au cours de la cryoglobulinémie mixte et du syndrome de Sjögren
}

La cryoglobulinémie mixte* est caractérisée par une vascularite systémique qui évolue par poussées pouvant s'étendre sur des dizaines d'années. La fréquence d'une atteinte hépatique au cours de cette affection est connue de longue date. Les virus hépatotropes responsables d'infection prolongée sont de bons candidats dans sa pathogénie. Le rôle du virus $B$, suggéré dans un travail ancien en raison de la prévalence élevée des marqueurs spécifiques dans un groupe de malades, n'a pas été confirmé ultérieurement. L'avènement de techniques fiables pour le diagnostic de l'hépatite $\mathrm{C}$ permet désormais d'explorer la prévalence de cette infection au cours de la cryoglobulinémie mixte. Plusieurs groupes italiens et français viennent de rapporter au total 35 observations de patients ayant une cryoglobulinémie mixte de type II (avec composant monoclonal) ou de type III (polyclonale) associée à une sérologie HCV (hepatitis $C$ vinus) positive [1-3]. La spécificité des techniques ELISA ou RIBA (recombinant immunoblotting assay) dans ces travaux pouvant être douteuse ou n'étant pas précisée, ils demandaient à être confirmés.

Le groupe de Ferri, à Pise, vient d'apporter des précisions épidémiologiques à partir de 52 patients dont 23 ont une cryoglobuline de type II et 29 de type III [4]. Vingt-huit patients (54\%) ont des anticorps anti-HCV dépistés par un test RIBA de $1^{\text {re }}$ génération. Simultanément, $50 \%$ ont des marqueurs du virus B. La prévalence d'anticorps dirigés contre le virus $\mathrm{C}$, étudiée chez 59 témoins atteints de lupus ou d'arthrite rhumatoïde, est nulle et affecte $5 \%$ d'un groupe de 20 patients atteints de syndrome de Sjögren. La prévalence des anticorps anti-HCV est de 1,2\% parmi les témoins normaux. Une biopsie hépatique a été effectuée chez 30 sur 52 patients et a montré une hépatite chro- nique active ou persistante. Les anticorps anti-HCV ont été décelés dans le cryoprécipité chez 7 sur 28 patients. Le même groupe a exploré plus avant 42 sur 52 patients, d'une part, à l'aide d'un test RIBA de $2^{e}$ génération (plus sensible) et rapporte la présence d'anticorps anti-HCV chez $90 \%$ d'entre eux ; et, d'autre part, en pratiquant une amplification par PCR : la présence d'ARN du HCV, c'est-à-dire une virémie positive, est détectable dans $86 \%$ des sérums [5].

Ces résultats suggèrent vivement que l'HCV joue un rôle pathogène dans la survenue d'une cryoglobulinémie mixte. Cette affection est bien plus fréquente dans le sud de la France et en Italie qu'en Europe du Nord. Sa prévalence accrue se calque sur une prévalence également accrue du virus de l'hépatite $\mathrm{C}$ dans ces régions. A distance de ces contrées, la concordance de cette association apparemment étroite reste à établir. Si celle-ci se vérifiait, le nombre des cryoglobulinémies dites essentielles serait considérablement réduit. Ces informations suggèrent aussi d'évaluer l'intérêt d'antiviraux actifs sur HCV chez ces patients, en particulier l'interféron (IFN) dont l'intérêt (et les limites) au cours de l'hépatite $C$ sont établis [6]. Fortuitement, les résultats à long terme du traitement par interféron de 21 patients attcints de cryoglobulinémie essentielle du type II viennent d'être rapportés [7] : tous avaient une mala-

\footnotetext{
"Le terme "conoglobulinémie " signifie la présence durable d'immunoglobulines séniques anormales qui précipitent réversiblement au froid. De telles onyoglobulines sont dites mixtes lorsqu'elles sont composées d'au moins deux immunoglobulines.

** La sinladénile est une inflammation des glandes salivaires sous-maxillaires aboutissant da un tarissement des sécrétions salivaires et à une sécheresse de la bouche, ou xérostomie. D'autres sécrétions exacrines peuvent être altérées, en particulier les sécrétions larymales, réalisant un syndrome sec ou syndrome de Gougerot-Sjögren.
}

die grave, évoluant depuis huit ans en moyenne, résistant à un traitement par immunosuppresseur ou corticoïdes. Une hépatopathie était connue chez un tiers des patients. Tous ont été traités par IFN- $\alpha 2 a$ (18 patients) ou IFN- $\beta$ (3 patients), à la posologie de 3 millions d'unités par jour pendant trois mois, poursuivie ultérieurement un jour sur deux. Une rémission clinique complète a été observée chez 16 patients (77\%), le plus souvent au cours du premier mois de traitement ; de plus, chez la totalité des malades ayant une hépatopathie, les transaminases se sont normalisées. Cette rémission s'est poursuivie chez 9 patients (43\%) pendant en moyenne 32 mois ; chez 5 d'entre eux, il n'y a pas eu rechute à l'arrêt du traitement (recul moyen : 25 mois). Des effets indésirables ont motivé l'arrêt du traitement chez 2 patients ; 4 ont présenté des signes d'évolutivité malgré l'IFN et 1 patient est décédé d'infarctus myocardique. Chez 5 patients enfin, seule une rémission clinique incomplète a été observée avec persistance des signes neurologiques.

Dans cette étude, la prévalence d'anticorps anti-HCV n'est pas mentionnée ; l'effet remarquablement favorable de l'IFN sur l'évolutivité des manifestations liées à la cryoglobulinémie pourrait résulter de son activité antivirale, chez des patients éventucllement infectés par HCV, ou d'un effet sur le système lymphoïde déjà mis à profit pour le traitement de divers syndromes lymphoprolifératifs.

Par ailleurs, la prévalence de l'atteinte des glandes salivaires au cours de l'infection par HCV vient d'être rapportée chez 28 patients ayant une hépatopathie spécifique [8] : 16 sur 28 , soit $57 \%$ des malades contre $5 \%$ des témoins, présentaient histologiquement une sialadénite** focale lymphocytaire, tout à fait identique à ce qui est 
observé au cours du syndrome de Gougerot-Sjögren. Seulement 10 sur 28 patients se plaignaient de xérostomie ${ }^{* *}$, aucun n'avait de symptôme oculaire mais 3 sur 6 avaient un tarissement sécrétoire lacrymal objectivé par le test de Schirmer. Le mécanisme de l'infiltrat lymphocytaire des glandes salivaires n'est pas connu : il pourrait relever d'un phénomène dysimmunitaire systémique lié à l'infection par le virus $\mathrm{C}$, d'une atteinte directe des glandes salivaires, voire de l'intégration du génome viral au sein des cellules mononucléées. Une co-infection par le virus d'Epstein-Barr n'a malheureusement pas été étudiée dans ce travail, alors même que sa fréquence a été soulignée tout récemment dans le syndrome de Gougerot-Sjögren [9]

D. C.

A. D.

1. Bambara LM, Biasi D, Caramaschi P, Car letto A, Pacor ML. Cryogyobulinaemia and hepatitis C virus (HCV) infection (letter). Clin Exp Rheumatol 1991 ; 9 : 96-7.

2. Casato M, Taliani G, Pucillo LP, Goffredo F, Lagana B, Bonomo L. Cryoglobulinemia and hepatitis C virus. Lancet 1991; 337(i) : 1047-8 3. Harle JR, Disdier P, Durand JM, et al. Cryo globulinémie mixte au cours de l'infection par le virus de l'hépatite C (10 cas). Presse Méd 1991 $20: 1233$.

4. Ferri C, Greco F, Longombardo G, et al Antibodies to hepatitis $\mathrm{C}$ virus in patients with mixed cryoglobulinemia. Arthritis Rhoum 1991 ; $34: 1606-10$.

5. Ferri C, Greco F, Longombardo G. Association between hepatitis $\mathrm{C}$ virus and mixed cryoglobulinemia. Clin Exp Rhewnalol $1991 ; 9: 621-4$ 6. Di Bisceglie AM, Hoofnagle JH. Therapy of chronic hepatitis $\mathrm{C}$ with alpha interferon. Hepalology $1991 ; 13$ : 601-3.

7. Casato M, Lagana B, Antonelli G, Dianzani F, Bonomo L. Long term results of therapy with interferon- $\alpha$ for type II essential mixed cryoglobulinemia. Blood 1991; 78 : 3142-7.

8. Haddad J, Deny P, Munz-Gotheil C, et al Lymphocytic sialadenitis of Sjögren's syndrome associated with chronic hepatitis $\mathrm{C}$ virus liver disease. Lancet $1992 ; 339$ : 321-3.

9. Mariette X, Gozlan J, Clerc D, et al. Detection of Epstein-Barr virus DNA by in situ hybridization and polymerase chain reaction in salivary gland biopsy specimens from patients with Sjögren's syndrome. Am J Med 1991; 90 Sjogren's

$\mathrm{m} / \mathrm{s} n^{\circ} 4$, vol. 8, auril 92

\section{口n BRÈVES $\square$}

Rôles respectifs de l'hyperproduction de l'interleukine 6 et d'un réarrangement chromosomique dans le plasmocytome murin. Il est possible de produire dans certaines lignées de souris (Balb/c et $N Z B$ ), des plasmocytomes expérimentaux par traitement avec des huiles minérales ou d'autres composés. Ces tumeurs sont caractérisées, chez les souris $\mathrm{Balb} / \mathrm{c}$, par une translocation chromosomique $\mathrm{t}(12$; 15) et par une hyperproduction d'interleukine 6 (IL6). Dans le myélome humain, l'hypersécrétion d'IL6 est également considérée comme un facteur de mauvais pronostic et comme l'un des mécanismes de l'hyperprolifération plasmocytaire [1]. Afin d'étudier les rôles respectifs de la translocation chromosomique et de l'hyperproduction d'IL6, des chercheurs japonais d'Osaka ont créé des souris transgéniques exprimant le gène de l'IL6 sous le contrôle des régions régulatrices d'un gène du complexe majeur d'histocompatibilité [2]. Sur le fonds génétique C57Bl/6, ces animaux développent une plasmocytose massive sans tumeur et sans production d'immunoglobulines monoclonales. En revanche, le passage de ce même transgène dans le fonds génétique Balb/c s'accompagne de l'apparition de plasmocytomes monoclonaux au niveau desquels on retrouve la translocation $\mathrm{t}(12 ; 15)$. Cet accident chromosomique, comme les translocations $t(6 ; 15)$ également observées dans les plasmocytomes expérimentaux de la souris, aboutissent à l'activation de $c$-myc, suggérant qu'une hyperproduction d'IL6 et une hyperexpression du gène myc coopèrent normalement pour produire le plasmocytome. D'ailleurs, de telles tumeurs peuvent également survenir chez des souris traitées par des agents inducteurs et surinfectées par un rétrovirus contenant le gène viral $v$-myc; dans ce cas, le gène $c-m y c$ endogène n'est pas réarrangé. Ces expériences démontrent également que, chez les souris transgéniques Balb/c, l'hypersécrétion d'IL6 peut avoir un rôle tout à fait important, déclenchant, dans la progression tumorale aboutissant à un plasmocytome. Il provoque une expansion polyclonale des plasmocytes à l'occasion de laquelle survient, sur certains terrains génétiques, une activation d'un gène myc par réarrangement chromosomique.

[1. Klein B, Bataille R. médecine/scien ces $1991 ; 7$ : 937-43.]

[2. Suematsu S, et al. Proc Natl Acad Sci USA 1992 ; 89 : 232-5.]

Origine paternelle de la délétion du chromosome 4 dans le syndrome de Wolf-Hirschhorn. Le syndrome de Wolf-Hirschhorn est une très grave affection, caractérisée notamment par un faible poids de naissance, un retard du développement, et des malformations telles que microencéphalie et fentes labiale et palatine. Il résulte d'une monosomie partielle du bras court du chromosome 4 et apparaît le plus souvent de façon sporadique, les parents étant cliniquement normaux. Deux travaux $[1,2]$ se sont attachés à déterminer l'origine parentale à l'aide de sondes de la région 4 p16.3. Ils portent respectivement sur 7 et 5 malades. Une origine paternelle a été reconnue dans tous les cas. Pour expliquer cette transmission, on a évoqué une empreinte parentale, renforcée par le fait que, dans la maladie de Huntington, l'affection est en général plus grave et apparaît plus tôt quand elle est héritée du père. Le bras court du chromosome 4 serait donc soumis au phénomène du sceau parental. Un argument toutefois s'oppose à cette hypothèse : il existe des formes familiales (10 à $15 \%$ des cas), dues à une translocation chromosomique chez un des parents. Or les deux tiers de ces cas reconnaissent une origine maternelle. Il n'est donc pas possible de penser qu'une délétion maternelle soit létale ou passerait inaperçue, et l'explication finale de la transmission pater nelle dans les formes sporadiques reste à découvrir.

[1. Quarrell OWJ, et al. J Med Gene 1991; 28 : 256-9.]

[2. Tupler R, et al. J Med Genet 1992 29 : 53-5.]

[3. Narahara K, et al. Jpn J Med Hum Genet 1984 ; 29 : 403-11 (in [1]).] 